\title{
Protein Interaction Mapping of Translational Regulators Affecting Expression of the Critical Stem Cell Factor Nos
}

\author{
Sumira Malik ${ }^{1,2}$, Wijeong Jang ${ }^{1}$ and ${ }^{\dagger}$ Changsoo Kim ${ }^{1}$ \\ ${ }^{1}$ Hormone Research Center, School of Biological Sciences and Technology, College of Natural Sciences, \\ Chonnam National University, Gwangju 61186, Republic of Korea \\ ${ }^{2}$ Dept. of Agriculture and Forestry, Tula's Institute Dhoolkot, Dehradun-248011, India
}

\begin{abstract}
The germline stem cells of the Drosophila ovary continuously produce eggs throughout the life- span. Intricate regulation of stemness and differentiation is critical to this continuous production. The translational regulator Nos is an intrinsic factor that is required for maintenance of stemness in germline stem cells. Nos expression is reduced in differentiating cells at the post-transcriptional level by diverse translational regulators. However, molecular mechanisms underlying Nos repression are not completely understood. Through three distinct protein-protein interaction experiments, we identified specific molecular interactions between translational regulators involved in Nos repression. Our findings suggest a model in which protein complexes assemble on the 3' untranslated region of Nos mRNA in order to regulate Nos expression at the posttranscriptional level.
\end{abstract}

Key words : Nos, Ovary, Oogenesis, Stem cells, Drosophila

\section{INTRODUCTION}

Female germline stem cells (GSCs) located in the anterior end of the Drosophila ovary continuously produce eggs throughout the life-span. In particular, GSCs attached to the somatic cap cells exhibit asymmetric cell division to generate a stem cell (self- renewal), which is also attached to the cap cells, and a cystoblast (CB), which is one cell away from the cap cells and enters a differentiation program (Spradling et al., 1997). The CB undergoes four incomplete divisions to generate a 16-cell cyst, one of which will be an oocyte and the others nurse cells.

Maintenance of GSCs is dependent on signals emanating from the cap cells, which construct a niche environ- ment supporting the GSCs. Decapentaplegic (Dpp) is a critical extrinsic niche signal that stimulates continued stemness of GSCs (Xie \& Spradling, 1998). In addition, intrinsic factors expressed in GSCs mediate stemness and block differentiation. The translational regulator Nanos (Nos) is one such intrinsic factor required for stemness maintenance (Lin \& Spradling, 1997; Forbes \& Lehmann, 1998). Nos collaborates with another translational regulator, Pumilio (Pum), to repress expression of factors that promote stem cell differentiation (Joly et al., 2013).

In contrast, Bag of marbles (Bam) is an intrinsic differentiation-promoting factor expressed in CBs (McKearin \& Spradling, 1990). In a bam mutant ovary, GSCs fail to undergo differentiation and produce stem cell-like tumor

\footnotetext{
Manuscript received December 22, 2017, Received in revised form December 26, 2017, Accepted December 27, 2017

† Corresponding Author : Changsoo Kim, Hormone Research Center, School of Biological Sciences and Technology, College of Natural Sciences, Chonnam National University, Gwangju 61186, Republic of Korea. Tel: +82-62-530-5201, E-mail: changgk2001@ hanmail.net

This is an Open Access article distributed under the terms of the Creative Commons Attribution Non-Commercial License (http:// creativecommons.org/licenses/by-nc/3.0) which permits unrestricted non-commercial use, distribution, and reproduction in any medium, provided the original work is properly cited.
} 
cells (McKearin \& Spradling, 1990). Upon ectopicexpression in GSCs, Bam promotes differentiation of stem cells (McKearin \& Ohlstein, 1995; Ohlstein \& McKearin, 1997). One role of Bam in differentiating cells is to repress Nos expression at the translational level through the nos 3' untranslated region (UTR) (Li et al., 2009). Other critical differentiation-promoting factors include Benign gonial cell neoplasm (Bgcn), Sex lethal (Sxl), and Meiotic P26 (Mei-P26) (Chau et al., 2009; Ohlstein et al., 2000; Li et al., 2013). Bgen encodes a protein distantly related to DExH-box RNA helicase, while Sxl is an RNA-binding protein with specificity to polyuridines stretches and MeiP26 is an RNA-binding protein with an NHL (Ncl-1, HT2A and Lin-41) domain that functions as a translational repressor (Sekelsky et al., 1999; Glasscock et al., 2005; Chau et al., 2012). Genetic studies suggest that Bam functionality requires $\mathrm{Bgcn}, \mathrm{Sxl}$, and Mei-P26, suggesting that Bam acts in conjunction with these proteins (Chau et al., 2012; Li et al., 2013). However, the mechanics of how Bam recognizes nos mRNAs and how it collaborates with Bgen, Sxl, and Mei-P26 remain poorly understood.

To understand the molecular mechanisms underlying Nos repression, we undertook rigorous protein-protein interaction studies and performed interaction mapping on Bam, Bgen, Sxl, and Mei-P26. In doing so, we identified two more translational regulators, Brain tumor (Brat) and Argonaute-1 (Ago1), which physically interact with Bam and Sxl. Our results suggest a model in which translational repressors are assembled on the cis-element of nos mRNAs to mediate translational regulation.

\section{MATERIALS AND METHODS}

\section{Chemicals and antibodies}

All chemicals were obtained from Sigma-Aldrich. Primary antibodies were obtained as follows; anti- FLAG (M2; Sigma) (mouse), anti-Myc (rabbit) (Cell Signaling), and anti-HA (rat) (Roche Applied Science). All secondary anti- bodies were obtained from Roche Applied Science.

\section{Vector constructions}

The S2 cell expression vectors, pAc5.1A-FLAG- Bam and pAc5.1A-Myc-Bgen were obtained from the previous studies (Kim et al., 2010). The NHL domain (aa 7241037), the N-terminus (aa 1-730), and the full-length of Brat (LD28374 from the Drosophila Genomics Resource Center) were cloned into the NotI/XbaI site of pAc5.1AMyc to generate pAC5.1A-Myc-Brat NHL and a pAC5. 1A-Myc-Brat $\mathrm{N}$ terminal, and the pAC5.1A-Myc-Brat FL tagged proteins in S2 cells. For yeast two-hybrid, the fulllength of Bam was cloned into Bam HI/XhoI sites of pLexA (Clontech) to generate LexA-Bam. The N- terminus and full length of Brat were cloned into the Bam HI/ SacI and Bam HI/XhoI, respectively, of pACT2 (Clontech) to generate TAD-Brat FL and -Brat N. The Brat NHL domain (aa 724-aa 1037) was cloned into SalI/Xba1 of pGAD 424 (Clontech) to generate TAD-Brat NHL. For the fragment complementation assay in HEK293T cells, the coding sequence and its derivatives of Bam and Brat were cloned into Bam HI/XhoI and the KpnI/HindIII site of the vectors (CoralHue_Fluo-chase kit; Medical and Biological Lab Co.), respectively, to produce protein fused to either the $\mathrm{N}$ - or the $\mathrm{C}$ - terminal fragment of the monomeric $\mathrm{Ku}$ sabira-Green (mKG). P50- mKG_N and P65-mKG_C were provided as the control.

\section{Yeast two-hybrid assays}

The various combinations of plasmids expressing the LexA DNA binding domain-fused protein and the GADfused protein were co-transformed into the yeast strain YPH500 (MAT $\alpha$, ade 2, his3, leu 2, lys2, trp1, ura3) harboring the pSH18-34 plasmid (lexAop- LacZ reporter) (Gyuris, Golemis et al., 1993) by the standard lithium acetate method (Ito et al., 1983). The independent transformants were patched onto glucose plates containing Xgal for 24-48 hours, followed by a beta-galactosidase 
liquid assay as described (Ponticelli et al., 1995).

\section{Co-immunoprecipitation assay}

The Drosophila Schneider's 2 cells (S2 cells) were maintained in a Shields and Sang M3 insect medium supplemented with $10 \%$ insect medium supplement (Sigma-Aldrich) and antibiotics (Hyclone) in a humidified atmosphere at $25^{\circ} \mathrm{C}$. S2 cells were transiently transfected with expression vectors, using the DDAB method (Han, 1996). Transfected cells harvested $72 \mathrm{~h}$ after transfection were washed in phosphate-buffered saline (Hyclone Dulbecco's Phosphate Buffer saline) and lysed with radioimmune precipitation assay buffer $(50 \mathrm{mM}$ Tris- $\mathrm{HCl}, \mathrm{pH} 8.0,150 \mathrm{mM}$ $\mathrm{NaCl}, 1 \%$ Nonidet P-40, $5 \mathrm{mM}$ EDTA, and $1 \mathrm{mM}$ phenylmethylsulfonyl fluoride; ELPIS-Biotech.) containing protease inhibitors. The lysates were clarified by centrifugation at 13,000 rpm (Eppendorf centrifuge) for $10 \mathrm{~min}$ at $4^{\circ} \mathrm{C}$. The cleared extracts $(3 \mathrm{mg})$ were mixed with $40 \mu \mathrm{L}$ of anti-FLAG M2- conjugated agarose beads (Sigma Aldrich) and rotated at $4^{\circ} \mathrm{C}$ overnight. The beads were precipitated by Eppendorf centrifugation and washed three times with washing buffer (20 mM HEPES (pH 7.7), $150 \mathrm{mM} \mathrm{NaCl}$, $2.5 \mathrm{mM} \mathrm{MgCl}_{2}, 0.05 \%$ Nonidet P-40, $10 \%$ glycerol, and 1 $\mathrm{mM}$ dithiothreitol) containing protease inhibitors. The bound proteins were eluted in $50 \mu \mathrm{L}$ of $0.1 \mathrm{mM}$ glycine acetate (pH 3.0) and precipitated by adding $10 \%$ (the final concentration) trichloroacetic acid and 1\% (final) sodium deoxycholate. The elutes were incubated $30 \mathrm{~min}$ at $-20^{\circ} \mathrm{C}$ and precipitated by centrifugation. The pellets were suspended in a 2XSDS loading buffer, and Western blot analysis was performed using anti-Flag and -Myc.

\section{Protein Fragment Complementation Analysis (FCA)}

HEK293T cells were transiently transfected with vectors to express the mKG_N and mKG_C fusion proteins. The cells were plated on an 8-well cell culture slide (SPL) and then allowed to grow in medium. Then $24 \mathrm{~h}$ after transfection, $100 \mu \mathrm{L}$ of $3.7 \%$ formaldehyde in 1XDPBS (Dulbe- cco's Phosphate Buffered Saline) was added to the cells for $5 \mathrm{~min}$. The cells were then washed with 1XDPBS twice and were mounted on a drop of prolong Gold (Invitrogen). Images of the cells were acquired by confocal microscopy. GFP signals from either $\mathrm{p} 50-\mathrm{mKG} \mathrm{N}$ or $\mathrm{p} 65-\mathrm{mKG} \mathrm{C}$ alone or from a combination of these two were used as negative and positive controls, respectively.

\section{RESULTS AND DISCUSSION}

\section{Bam physically interacts with Mei-P26 and Brat}

Genetic studies have shown that Bam is a critical factor in Nos repression and that its function requires Bgen, MeiP26, and Sxl (Chau et al., 2012; Li et al., 2013). However, individual interactions between them were not fully defined. We employed three different approaches to define these interactions.

To examine whether Bam physically interacts with Mei-P26, we performed a yeast two-hybrid assay (Kim et al., 2010). A LexA DNA binding domain (LexA) was fused to Bam full-length (FL) protein (LexA-Bam FL), while the transcriptional activation domain (TAD) of the Gal4 protein was fused to Mei- P26 FL protein. Transfection of yeast cells with LexA- Bam FL and TAD-Mei-P26 FL revealed that Bam physically interacted with MeiP26. As Mei-P26 contains an NHL domain at its C-terminus, we tested whether that domain mediates interaction with Bam. Assays with the C-terminal NHL domain (Mei-P26 NHL) and the N-terminus (Mei-P26 N) lacking the NHL domain revealed that Bam interacted with the N- terminus (1-920), but not the NHL domain (921-1140) of Mei-P26 (Fig 1A).

Like Mei-P26, Brat is a member of the NHL domain protein family, is expressed in differentiating cells, and promotes stem cell differentiation (Harris et al., 2011), raising the possibility that Brat might function together with Bam in Nos repression. Thus, we performed a yeast two-hybrid assay to determine if Bam interacts with Brat, 

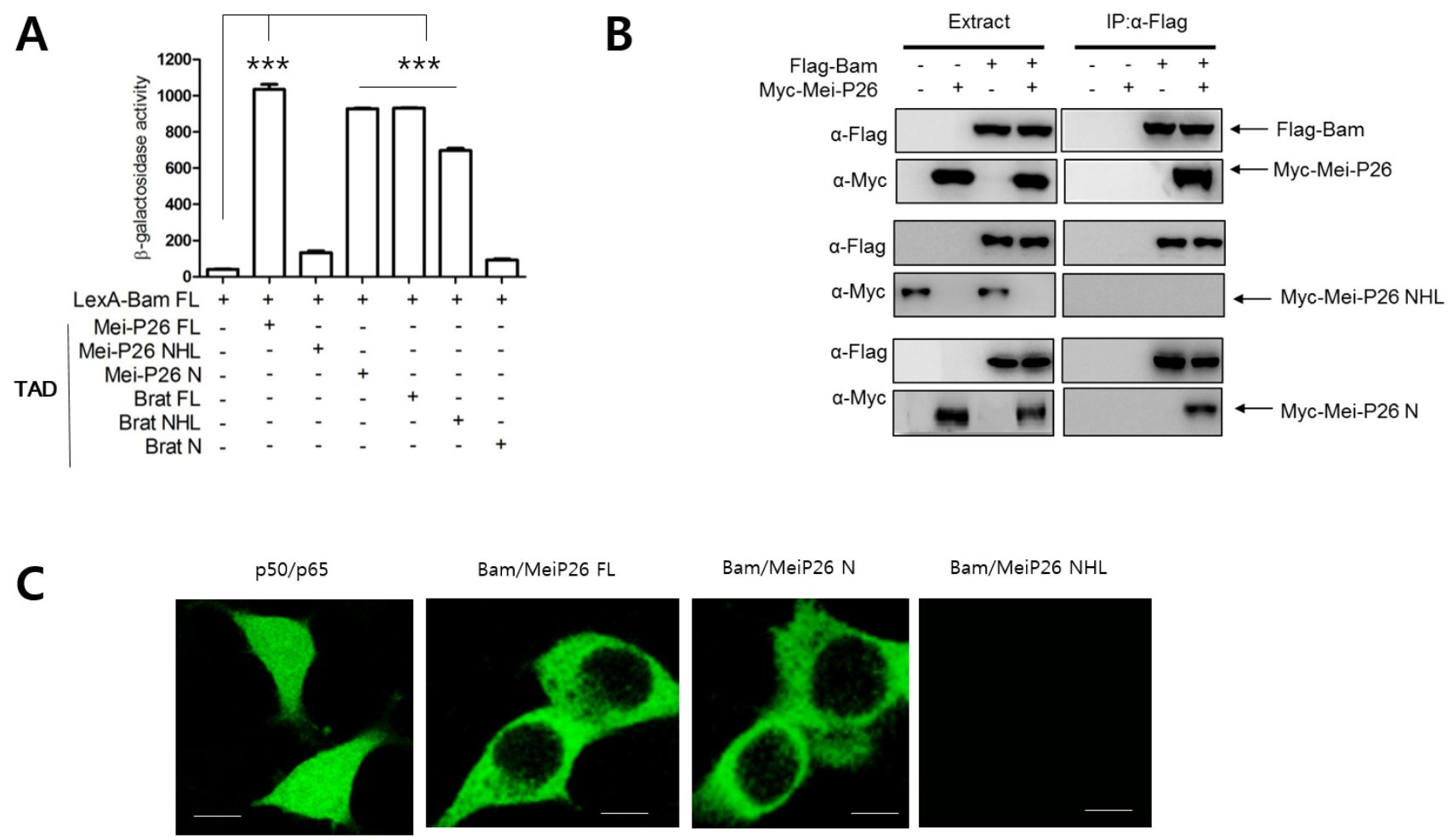

Bam/MeiP26 NHL

Fig. 1. Bam physically interacts with Mei-P26 and Brat. (A) Yeast two-hybrid assay, with protein-protein interactions monitored using $\beta$-galactosidase assays. Bam full- length sequence was fused with the LexA DNA-binding domain (denoted as LexA-Bam FL). Other constructs were fused to the Gal4 transcriptional activation domain (TAD). FL, N, NHL indicates full-length, the N-terminus, and the C-terminal NHL domain of Mei-P26 or Brat. Mean \pm SD values were obtained from three independent experiments. $P$ values were determined by Student's $t$-test in Sigma Plot. ${ }^{* * *}$ $P<0.001$. (B) Pull-down assay. S2 cell lysates co-expressing Flag-Bam with Myc-Mei-P26 FL, Myc-Mei-P26 N, and Myc-Mei-P26 NHL were subjected to immunoprecipitation with Flag antibody ( $\alpha$-Flag). The extract (1/10 of the lysate) and the resulting immunoprecipitates (IPs) were subjected to immunoblot analysis with Flag and Myc antibodies. Three independent experiments were carried out with similar results. (C) Fragment complementation assay. Confocal images of HEK293 cells expressing proteins fused to either N-terminal (GFP-N) or C-terminal (GFP-C) fragments of GFP. P50/p65 was used as a positive control, with p50 fused to GFP-N and p65 fused to GFP-C. For assaying Bam/Mei-P26 FL, Bam/Mei-P26 N, and Bam/Mei-P26 NHL, Bam was fused to GFP-N and others fused to GFP-C. Three independent experiments were carried out with similar results. The scale bar indicates $10 \mu \mathrm{m}$.

and found that LexA-Bam interacted with TAD-Brat FL. Unlike Mei-P26, LexA-Bam interacted with Brat NHL (724-1,037), but not the Brat N-terminus (1-730) (Fig. 1A).

We further performed pull-down assays to confirm the yeast two-hybrid results. Bam was fused to a Flag tag (Flag-Bam), while MeiP26 and its derivatives were fused to Myc tags (Myc-Mei-P26, Myc-Mei-P26 NHL and MycMei-P26 N). Flag-Bam was then expressed with Myctagged proteins in S2 cells, and immunoprecipitation of S2 cell lysates performed with Flag antibodies. The Flag-Bam precipitates were subjected to western blot with Flag and Myc antibodies. Flag-Bam precipitates contained MycMei-P26 and Myc-Mei-P26 N, but not Myc-Mei-P26 NHL (Fig. 1B), supporting results from the yeast two-hybrid assays. We conclude that that Bam physically interacts with the N-terminus of Mei-P26. Similar experiments were performed with Flag-Bam and Myc-Brat or Myc-Brat derivatives, confirming that Bam interacted with Brat through 
the Brat NHL domain (Table 1).

To further confirm our findings, we carried out GFP fragment complementation assays in mammalian cells (Kim et al., 2010). Bam was fused to an N-terminal fragment of GFP (Bam-GFP-N), while Mei-P26 and its derivatives were fused to the corresponding C-terminus fragment (Mei- P26GFP-C, Mei-P26 N-GFP-C, and Mei-P26-NHL-GFP-C). Confocal microscopic observation revealed GFP fluorescence when Bam-GFP-N and Mei-P26 GFP-C fragments were co-expressed in cells. Neither single Bam-GFP-N nor single Mei-P26-GFP-C expression revealed GFP fluorescence (Fig. 1C). Bam-GFP-N and Mei-P26 N GFP-C, but not Mei-P26 NHL, did produce GFP fluorescence (Fig. 1C). Similar experiments were conducted with Bam-GFP$\mathrm{N}$ and Brat-GFP-C, Brat N- GFP-C, or Brat-NHL-GFP-C fragments, confirming that Bam interacted with the Brat N-terminus, but not Brat NHL.

\section{Sxl physically interacts with Brat and Mei-P26}

Like Bam, Sxl is a critical differentiation-promoting factor and represses Nos expression through interaction with the nos 3'UTR (Chau et al., 2012). Genetic analysis

Table 1. Protein-protein interaction

\begin{tabular}{ccccc}
\hline \hline & Bam & Bgcn & Ago-1 & Sxl \\
\hline Mei-P26 FL & $+++^{1,2,3}$ & $+++^{1}$ & $-^{1},+^{3}$ & $++++^{1,2}$ \\
Mei-P26 NHL & $-^{1,2}$ & $+++^{1}$ & $-{ }^{1}$ & $++^{1}$ \\
Mei-P26 N & $+++^{1,2,3}$ & - & - & - \\
Brat FL & $+++^{1,2,3}$ & ND & $+++^{1,3}$ & $+++^{1,2}$ \\
Brat NHL & $+++^{1,2,3}$ & ND & - & $++++^{1,3}$ \\
Brat N & $-{ }^{2,3}$ & ND & $+++^{1}$ & $+++^{1,3}$ \\
Bam & ND & ND & $-{ }^{3}$ & - \\
Sxl & $-{ }^{1,3}$ & $-1,2$ & -3 & ND \\
\hline
\end{tabular}

\footnotetext{
${ }^{1}$ Yeast two-hybrid assay, ${ }^{2}$ Pull-down assay, ${ }^{3}$ GFPfragmentassay
}

FL, NHL, N denotes full-length, NHL domain, N-terminus. has shown that Bam requires $\mathrm{Sxl}$ for Nos repression ( $\mathrm{Li}$ et al., 2013). We carried out yeast two-hybrid assays with TAD-Sxl and LexA-fused proteins, and found that Sxl did not interact with Bam or Bgen (Fig. 2A). However, Sxl did interact with Brat and Mei-P26 (Fig. 2A). In particular, Sxl interacted with the C-terminal NHL domain but not the Nterminus of Mei-P26, and with both the Brat N-terminus and the Brat NHL domain. In pull-down assays, Flag-Sxl co-precipitated with Myc-Brat, Myc-MeiP26, and MycBgen.

\section{Model illustrating protein assembly on nos 3'UTR}

Our protein-protein interaction assays revealed a detailed map of interactions between Bam, Bgcn, Brat, MeiP26, Sxl, and Ago1 (Fig. 3A). Based on this map, we propose a model illustrating how these proteins are assembled on the nos 3'UTR (Fig 3B). The model suggests that Sxl recognizes the distal part (781-850 3'UTR) of nos mRNAs, while Ago1-microRNA complexes recognize the proximal region (1-100 3'UTR). These two regions have been previously shown to mediate Nos repression (Li et al., 2009; Chau et al., 2012). Ago1 and Sxl recruit Brat, which in turn recruits Bam, Bgcn, and Mei-P26. These repressive complexes would mediate either translational repression or mRNA decay. Further research is needed to determine whether Agol and Sxl bind the proximal or distal regions of the nos 3'UTR, and whether binding of other translational regulators (Bam, Bgen, Brat, Mei-P26) is dependent on Agol and Sxl. Moreover, it has yet to be determined whether Agol and Brat participate in Nos repression in vivo.

\section{ACKNOWLEDGEMENTS}

We thank the Drosophila Genetic Resource Center for providing cDNAs. This research was supported by the Basic Science Research Program through the National Research Foundation (NRF) of Korea, funded by the Ministry of Science, ICT and Future Planning (Grant No. 2015R1A2 
A

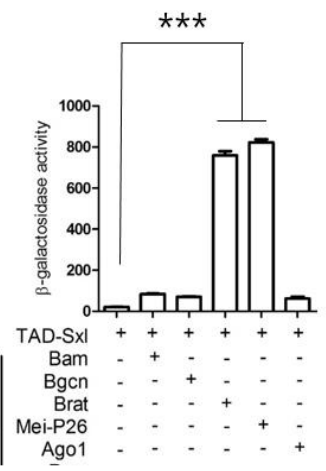

C

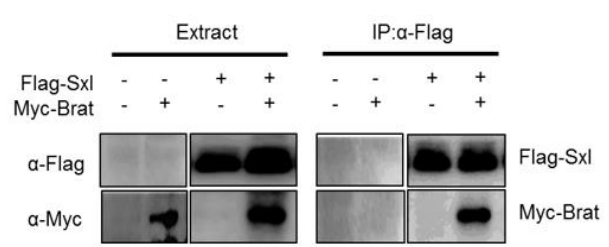

B

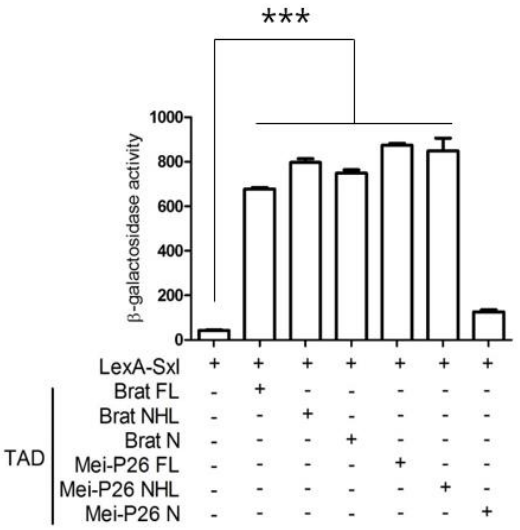

D

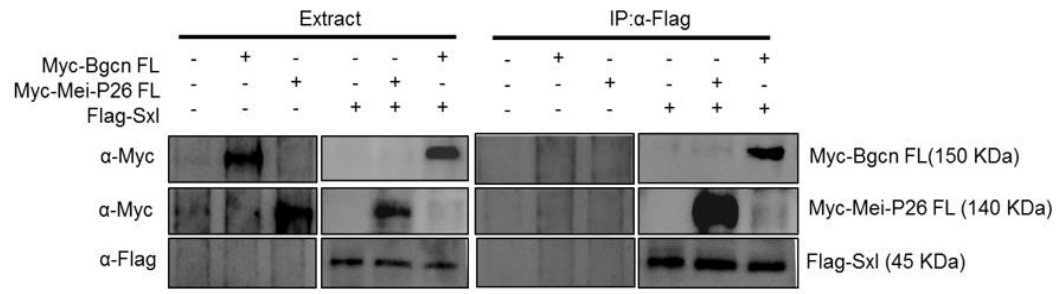

Fig. 2. Sxl physically interacts with Mei-P26, Brat, Pum, and Bgen. (A, B) Yeast two-hybrid assay, with protein-protein interactions monitored by $\beta$-galactosidase assays. (A) Sxl full- length sequence was fused with the transcriptional activation domain (TAD) of Gal4 (TAD-Sxl). The other constructs were fused to the LexA DNA-binding domain (LexA). (B) Sxl full-length sequence was fused with the LexA DNA-binding domain (LexA-Sxl). The other constructs were fused to the TAD of Gal4. FL, N, NHL respectively indicate full-length, N-terminus, and the C-terminal NHL domain of Mei-P26 or Brat. Mean \pm SD values were obtained from three independent experiments. $P$ values were obtained by Student's $t$-test in Sigma Plot. ${ }^{* * *} P<0.001$. (C, D) Pull-down assay. (C) S2 cell lysates coexpressing Flag-Sxl with Myc-Brat were subjected to immunoprecipitation with Flag antibody ( $\alpha$-Flag). The extract $(1 / 10)$ and the resulting immunoprecipitates (IPs) were subjected to immunoblot analysis with Flag and Myc antibodies. (D) S2 cell lysates co-expressing Flag-Sxl with Myc-Bgcn FL (upper) and Myc- Mei-P26 FL (lower) were subjected to immunoprecipitation with Flag antibody. FL denotes full-length. The extract (1/10) and the resulting IPs were subjected to immunoblot analysis with Flag and Myc antibodies.

A2A01004803).

\section{REFERENCES}

Chau J, Kulnane LS, Salz HK (2009) Sex-lethal facilitates the transition from germline stem cell to committed daughter cell in the Drosophila ovary. Genetics 182: 121-132.

Chau J, Kulnane LS, Salz HK (2012) Sex-lethal enables germline stem cell differentiation by down-regulating
Nanos protein levels during Drosophila oogenesis. Proc Natl Acad Sci USA 109:9465-9470.

Forbes A, Lehmann R (1998) Nanos and Pumilio have critical roles in the development and function of Drosophila germline stem cells. Development 125:679-690.

Glasscock E, Singhania A, Tanouye MA (2005) The MeiP26 gene encodes a RING finger B-box coiled-coilNHL protein that regulates seizure susceptibility in Drosophilia. Genetics 170:1677-1689.

Gyuris J, Golemis E, Chertkov H, Brent R (1993) Cdi1, a 

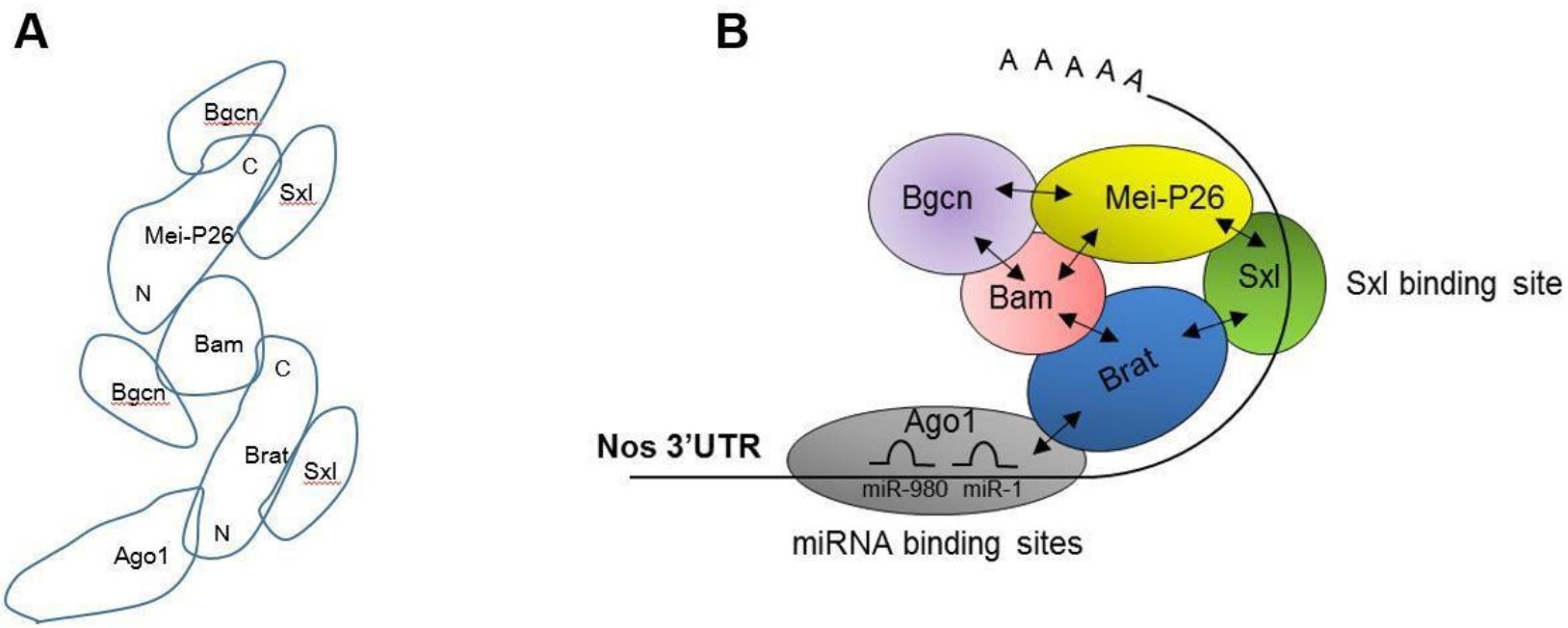

Fig. 3. Model illustrating protein assembly on nos 3'UTR. (A) Schematic drawing of a map of interactions revealed by our protein-protein interaction assays. (B) A model illustrating how these proteins are assembled on the nos 3'UTR.

human G1 and S phase protein phosphatase that associates with Cdk2. Cell 75:791-803.

Han K (1996) An efficient DDAB-mediated transfection of Drosophila S2 cells. Nucleic Acids Res 24:4362-4363.

Harris RE, Pargett M, Sutcliffe C, Umulis D, Ashe HL (2011) Brat promotes stem cell differentiation via control of a bistable switch that restricts BMP signaling. Dev Cell 20:72-83.

Ito H, Fukuda Y, Murata K, Kimura A (1983) Transformation of intact yeast cells treated with alkali cations. J Bacteriol 153:163-168.

Joly W, Chartier A, Rojas-Rios P, Busseau I, Simonelig M (2013) The CCR4 deadenylase acts with Nanos and Pumilio in the fine- tuning of Mei-P26 expression to promote germline stem cell self-renewal. Stem Cell Reports 1:411-424

Kim JY, Lee YC, Kim C (2010) Direct inhibition of Pumilo activity by Bam and Bgen in Drosophila germ line stem cell differentiation. J Biol Chem 285:47414716.

Li Y, Minor NT, Park JK, McKearin DM, Maines JZ (2009) Bam and Bgen antagonize Nanos-dependent germ-line stem cell maintenance. Proc Natl Acad Sci U SA 106:9304-9309.
Li Y, Zhang Q, Carreira-Rosario A, Maines JZ, McKearin DM, Buszczak M (2013) Mei-p26 cooperates with Bam, Bgen and Sxl to promote early germline development in the Drosophila ovary. PLoS One 8:e58301.

Lin H, Spradling AC (1997) A novel group of Pumilio mutations affects the asymmetric division of germline stem cells in the Drosophila ovary. Development 124: 2463-2476.

McKearin D, Ohlstein B (1995) A role for the Drosophila bag-of-marbles protein in the differentiation of cystoblasts from germline stem cells. Development 121: 2937-2947.

McKearin DM, Spradling AC (1990) bag-of-marbles: A Drosophila gene required to initiate both male and female gametogenesis. Genes Dev 4:2242-2451.

Ohlstein B, Lavoie CA, Vef O, Gateff E, McKearin DM (2000) The Drosophila cystoblast differentiation factor, benign gonial cell neoplasm, is related to DExH-box proteins and interacts genetically with bag-of-marbles. Genetics 155: 1809-1819.

Ohlstein B, McKearin D (1997) Ectopic expression of the Drosophila Bam protein eliminates oogenic germline stem cells. Development 124: 3651-3662.

Ponticelli AS, Pardee TS, Struhl K (1995) The glutamine- 
rich activation domains of human Sp1 do not stimulate transcription in Saccharomyces cerevisiae. Mol Cell Biol 15:983-988.

Sekelsky JJ, McKim KS, Messina L, French RL, Hurley WD, Arbel T, Chin GM, Deneen B, Force SJ, Hari KL, Jang JK, Laurencon AC, Madden LD, Matthies HJ, Milliken DB, Page SL, Ring AD, Wayson SM, Zimmerman CC, Hawley RS (1999) Identification of novel Drosophila meiotic genes recovered in a P-element screen. Genetics 152:529-542

Spradling AC, de Cuevas M, Drummond-Barbosa D, Keyes L, Lilly M, Pepling M, Xie T (1997) The Drosophila germarium: stem cells, germ line cysts, and oocytes. Cold Spring Harb Symp Quant Biol 62:25-34.

Xie T, Spradling AC (1998) Decapentaplegic is essential for the maintenance and division of germline stem cells in the Drosophila ovary. Cell 94:251-260. 